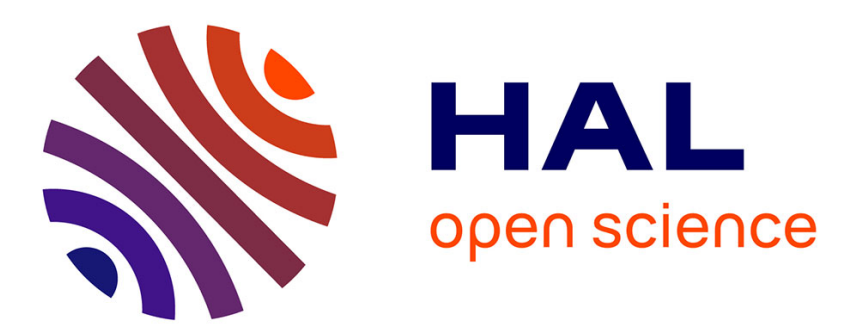

\title{
Exploring protein-protein interactions with large differences in protein expression levels using FLIM-FRET
}

\author{
Julien Godet, Yves Mely
}

\section{- To cite this version:}

Julien Godet, Yves Mely. Exploring protein-protein interactions with large differences in protein expression levels using FLIM-FRET. Methods and Applications in Fluorescence, 2019, 8 (1), pp.014007. 10.1088/2050-6120/ab5dd2 . hal-03034866

\section{HAL Id: hal-03034866 https://hal.science/hal-03034866}

Submitted on 1 Dec 2020

HAL is a multi-disciplinary open access archive for the deposit and dissemination of scientific research documents, whether they are published or not. The documents may come from teaching and research institutions in France or abroad, or from public or private research centers.
L'archive ouverte pluridisciplinaire HAL, est destinée au dépôt et à la diffusion de documents scientifiques de niveau recherche, publiés ou non, émanant des établissements d'enseignement et de recherche français ou étrangers, des laboratoires publics ou privés. 


\title{
Exploring protein-protein interactions with large differences in protein expression levels using FLIM-FRET
}

\author{
Julien Godet ${ }^{1,2 \bowtie}$ and Yves Mély ${ }^{1 凶}$ \\ ${ }^{1}$ Laboratoire de Bioimagerie et Pathologies, UMR 7021 CNRS, Université de Strasbourg, Faculté de pharmacie, Illkirch, France \\ ${ }^{2}$ Groupe Méthode Recherche Clinique, Pôle de Santé Publique, Hôpitaux Universitaires de Strasbourg, Strasbourg, France
}

\begin{abstract}
Many molecular processes within a cell are carried out by molecular machines built from a large number of proteins organized by their protein-protein interactions (PPIs). Exploring PPIs in their cellular context is critical to better understand the protein function. Förster resonance energy transfer measured by fluorescence lifetime imaging (FLIM-FRET) enables to monitor PPIs and to map their spatial organization in a living cell with high spatial and temporal specificity. But both the accurate measurement and the interpretation of multi-exponential FLIM-FRET data associated to mixtures of interacting and non-interacting proteins are difficult. Here we show that a simple diagram plot can find interesting visualization properties by clustering pixels with similar decay signatures. FLIM diagram plot can be used to provide valuable information about stoichiometry and binding mode in PPIs, even in the presence of large differences in protein expression levels of the different interacting partners. The proposed FLIM diagram plot is a useful visual approach for a more straightforward interpretation of complex lifetime data. This approach was applied for revealing critical features of PPIs in live Pseudomonas aeruginosa.

FLIM | Image analysis | Quantitative imaging | Bacteria | Pseudomonas aerug inosa

Correspondence: julien.godet @unistra.fr, yves.mely @unistra.fr
\end{abstract}

\section{Introduction}

Many molecular processes within a cell are carried out by molecular complexes built from a large number of proteins organized by their protein-protein interactions (PPIs) (1). PPIs play diverse roles and differ based on protein composition, affinity and whether the association is stable or transient (2). Exploring PPIs in their cellular context is a critical step to better understand the biological function of proteins.

Förster resonance energy transfer measured by fluorescence lifetime imaging (FLIM-FRET) enables monitoring PPIs and mapping their spatial organization in a living cell with high spatial and temporal specificity (3). FLIM is a method of choice to detect FRET in order to identify PPIs (4-10) including a recent report of high content screening assays (11). FLIM-FRET measures the fluorescence decay of a FRET donor at each pixel of the FLIM image. The fluorescence lifetime is a spectroscopic property of a fluorochrome sensitive to its chemical-physical environment. In the presence of a fluorescence acceptor in the close vicinity of a fluorescence donor, FRET can occur - increasing the non-radiative de-excitation rate $k_{n r}$ of the donor and thus decreasing its fluorescence lifetime. Because of the extreme distance dependence of FRET and the relatively large size of fused fluorescent proteins, two labelled proteins that undergo FRET have to physically interact. In contrast to intensity measurements, fluorescence lifetimes do not depend on excitation intensity nor fluorophore concentration. They are also poorly affected by photo bleaching so that FLIM unambiguously and directly report quantitatively on PPIs. This makes fluorescence lifetime approaches interesting for bioassays with application opportunities in biomedical and life science areas (12)

FLIM-FRET can additionally reveal sub-populations in a mixture of protein complexes. A fluorescence decay sums photons emitted from the different species present in the excitation volume. The resulting fluorescence decay is multiexponential and it is theoretically possible to extract distinct lifetimes and amplitudes of the different species composing the mixture. FLIM methods have generally been limited to mixtures of two species with mono-exponential decays as lifetime imaging of biological samples hardly provides the data quality and photons counts required for higher resolution. Even limited to two species, reasonably accurate values can be obtained for the mean lifetime $\langle\tau\rangle$, but not necessarily for the lifetimes and amplitudes of the individual components (13). To obtain robust fitting parameters for bi-exponential decay, it is recommended to use global analysis with a prior knowledge that only a limited number of fluorescent molecule species whose lifetimes do not vary spatially are present in the sample (14-16). Some efforts for alternative analysis (17), like phasor based analysis $(18,19)$ are also available, including attempts for denoising FLIM datasets and reducing bias and uncertainty in parameters estimations $(20,21)$. In the particular context of PPIs, non-interacting species (donor only decay) or interacting proteins where donors and acceptors are too far apart to undergo FRET can be discriminated from interacting ones in bi-molecular interactions assuming for example spatial invariance of the donor lifetime components across the data set. However the amplitude associated to the interacting species can be difficult to interpret notably as the consequence of large differences and variability in protein expression.

Here, we show that a simple diagram plot of the shortest lifetime component as a function of its amplitude and a subsequent spatial density analysis can report on the variability of 

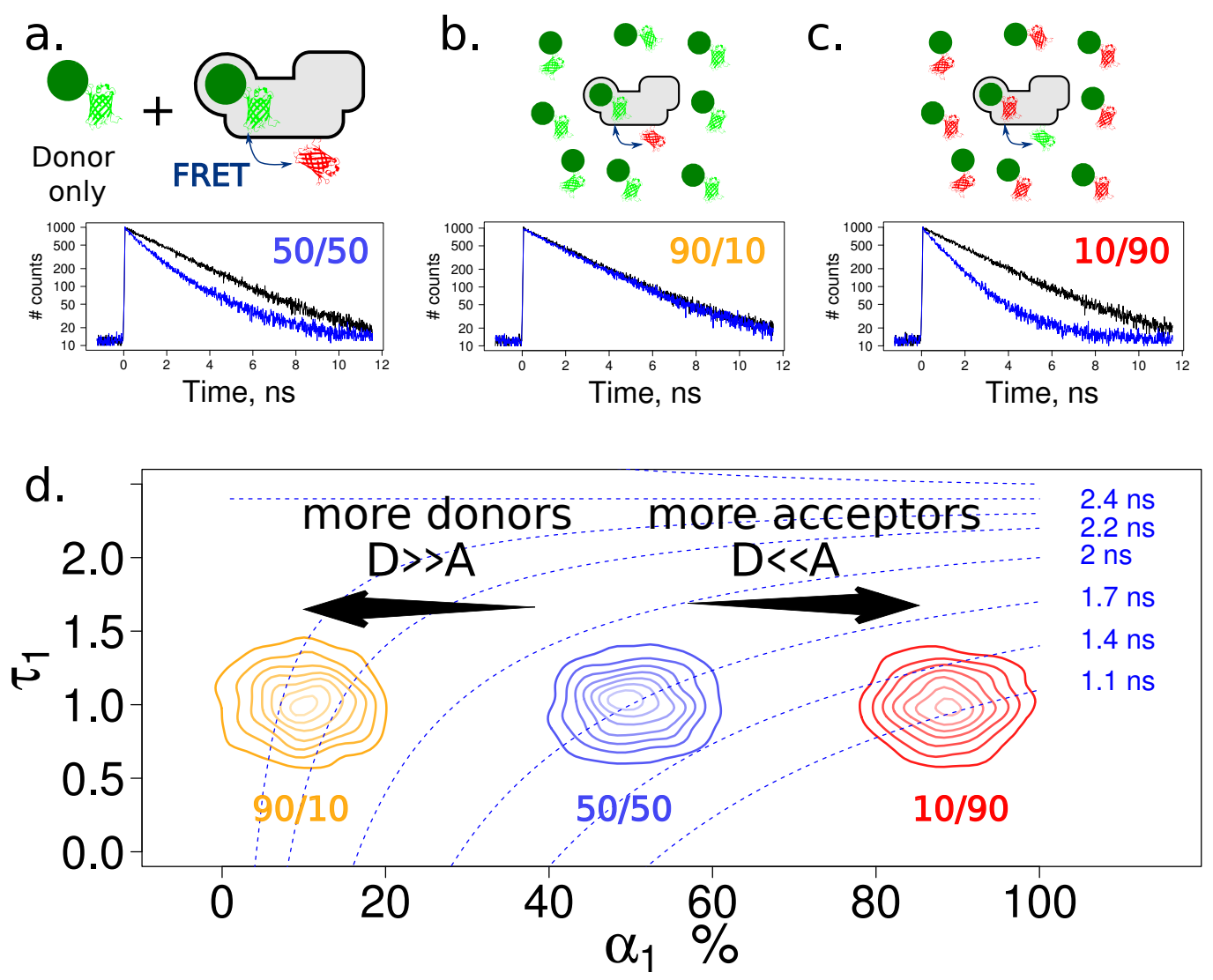

Fig. 1. PPIs and FLIM data visualized by FLIM diagram

a) Donor-labelled protein involved in a PPI may coexist in cell as a complex with its partner protein and as a free form. Both forms of energy-transferring and non-transferring donor-labelled proteins contribute to the donor fluorescence decay so that the recorded decay (blue) can significantly differ from the decay of the free donor (black), enabling FLIM to evidence PPI.

b) If many more proteins labelled with the fluorescence donor are present, the contribution of the numerous donors that do not undergo FRET will dominate the collected photons and the decay integrating the PPI will be very similar to the decay of the free donor (black - almost superimposed).

c) If the labelling scheme is reversed, the presence of numerous acceptors ensures that the proportion of free donors is comparatively low and the observed decay corresponds mostly to the decay of the complex.

d) The FLIM diagram is a simple plot of the shortest lifetime component $\tau_{1}$ as a function of its amplitude $\alpha_{1}$ defined for all the fitted pixels of a FLIM image. This plot allows clustering pixels with similar double exponential decay signatures. This visual representation of the data can report on the variability of $\tau_{1}$ and $\alpha_{1}$ parameters in the FLIM image. As seen on simulated noisy data with decays composed of a mixture of non-interacting species $\left(\tau_{2}=2.4 \mathrm{~ns}\right)$ and different fractions of interacting species $\left(\tau_{1}=1.0\right.$ $\mathrm{ns}$ ), the positions of the scatter plot are significantly changed. Large differences in protein expression can have a strong influence on $\alpha_{1}$ value that cannot therefore be directly interpreted as the fraction of complex present in the mixture.

the individual pixel parameters in the FLIM image. This diagram plot allows clustering pixels with similar double exponential decay signatures. Coupled to an experimental procedure consisting of recording decays of the two protein partners labelled with donor-acceptor and acceptor-donor successively, it can be used to provide critical information about stoichiometry and binding mode of PPIs. We applied this FLIM diagram for revealing key features of PPIs in a bacterial machinery involved in the bio-synthesis of the pyoverdine siderophore in Pseudomonas aeruginosa.

\section{FLIM diagrams}

The most popular approach for the fluorescence lifetime determination consists in using a least-square fitting analysis at each pixel or each group of binned pixels in the image. In a binding equilibrium mixture, two populations may contribute to the donor decay profile with one component arising from non-transferring molecules (unquenched donor in the free form) and one from molecules with inter-dye distances compatible with significant dipole-dipole energy transfer and usually associated to the bound form. A mixture of transferring and non-transferring fluorescent donors with intrinsic mono-exponential decays generates double-exponential decays (Figure 1). The mean lifetime is defined as a weighted sum of two decays according to:

$$
\langle\tau\rangle=\sum_{i=1}^{2} \alpha_{i} . \tau_{i}
$$

The fluorescence lifetime of the donor in the complex and its fraction relative to the fraction of free donors can be reasonably retrieved assuming a spatially invariant fluorescence lifetime for the free unquenched donor (see Supplementary Figure S1). To limit over-fitting and to improve fitting convergence, the long-lived lifetime $\tau_{2}$ is usually fixed at the value of the donor only fluorescence lifetime $(10,22,23)$, whereas the lifetime value of the fast decay $\tau_{1}$ and the relative contribution of each component defined by $\alpha_{1}$ and $\alpha_{2}$ 
are allowed to float. For a two-component model the amplitudes of both components are linked $\left(\alpha_{2}=1-\alpha_{1}\right)$; so that $\langle\tau\rangle$ can thus be rewritten as:

$$
\langle\tau\rangle=\tau_{2}-\alpha_{1} \times\left(\tau_{2}-\tau_{1}\right)
$$

A successful fit procedure will converge toward the couple of ( $\tau_{1}, \alpha_{1}$ ) values that adjusts the best to the decay conditionally to the $\tau_{2}$ value. The limited number of photons and the noise in the decays are responsible for the high variability of $\tau_{1}$ and $\alpha_{1}$ parameters as partial compensations can occur due to the large variability of these two parameters. If the estimation of $\langle\tau\rangle$ values are robust, the $\left(\tau_{1}, \alpha_{1}\right)$ couple solutions can be sub-optimal.

Plotting $\tau_{1}$ as a function of $\alpha_{1}$ is a simple way to explore observations made over all the fitted pixels. Although noisy, the scatter plot of $\left(\tau_{1}, \alpha_{1}\right)$ point is informative about the best estimation of these parameters. This estimation can be performed by calculating the spatial $2 \mathrm{D}$ density of the points to obtain the bivariate distribution of $\left(\tau_{1}, \alpha_{1}\right)$ parameters. The position of the centre of the scatter plot is informative about the fluorescence lifetime of the transferring donor and its associated relative amplitude. More than the estimated position, it provides $\tau_{1}$ and $\alpha_{1}$ conditional probability distributions given the externally determined and fixed $\tau_{2}$ value. Contour lines of $\langle\tau\rangle$ values can be added to the plot (dashedblue lines on Figure $1 \mathrm{~d}$ ) to facilitate data interpretation. Even by exploiting genetically expressed fluorescent proteins with mono-exponential decays, inhomogeneous cellular distribution of proteins and large differences in the protein expression levels of donor- and acceptor-labelled proteins may significantly modify the fluorescence decay. For example, in a situation in which a local abundance of free donors is observed in the sample, the contribution of the numerous donors that do not undergo FRET will dominate in the collected decay (Figure 1 b.). The more the excess of donors, the more the decay will tend similar toward the decay of the donor only as the fraction $\alpha_{1}$ is getting smaller (Figure $1 \mathrm{~d}$.). On the other hand, a large excess of acceptors relative to the amount of donors ensures that most of the donors are transferring. Indeed, as the excess of acceptors does not contribute to the signal (assuming the absence of direct excitation and / or the use of appropriate filters set), the decay tends to a mono-exponential decay with a lifetime $\tau_{1}$ (Figure $1 \mathrm{c}$. and d.). Therefore, without knowing the stoichiometry and the relative amount of transferring and non-transferring donors, $\alpha_{1}$ cannot be directly interpreted as the fraction of complex present in the mixture. Resolving the fraction of transferring species in cells is therefore complicated as the protein expression levels of donors and acceptors can strongly vary (24). Large differences in the protein expression levels of donors and acceptors are frequently observed in cells but are poorly reported as such in FLIM experiments. In a recent work, Gasser et al. (25) characterized the interaction of PvdA with PvdJ in live Pseudomonas aeruginosa. PvdA is an ornithine hydroxylase (26) working in tandem with the hydroxy-ornithine transformylase PvdF (27) to gen-

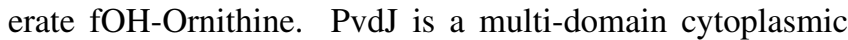
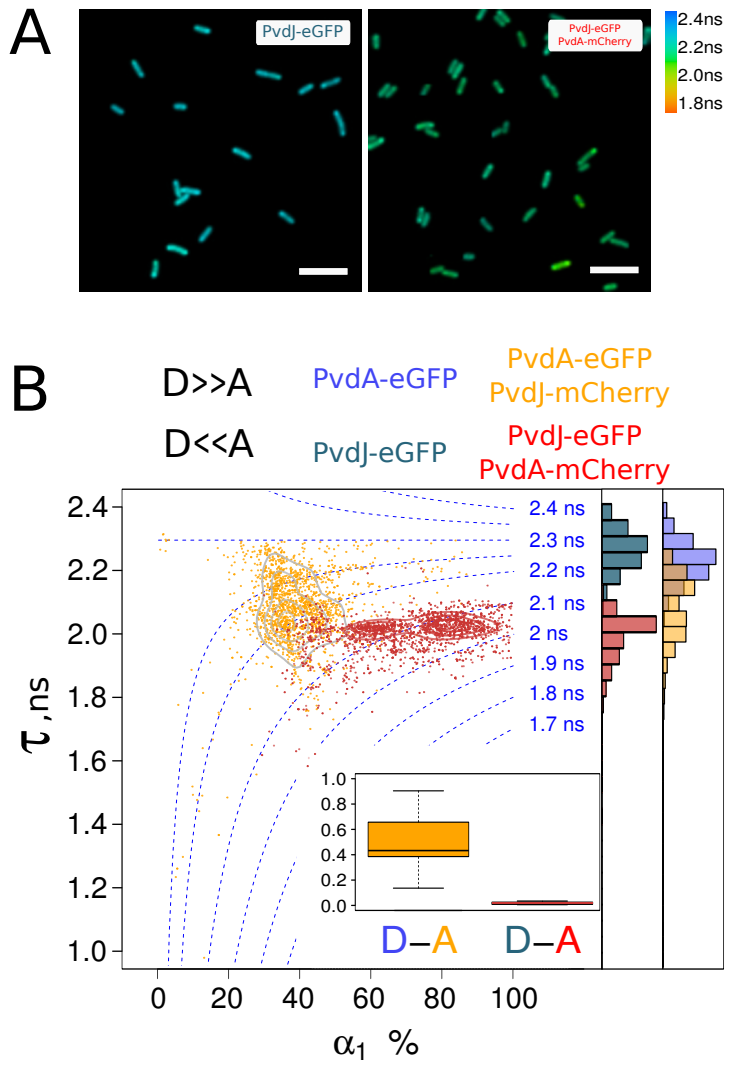

Fig. 2. FLIM images and FLIM diagrams of PvdA/PvdJ interactions A Representative FLIM images of PvdJ-eGFP and PvdJ-eGFP PvdA-mCherry $\mathrm{PAO} 1$ strains showing homogeneous shortened eGFP lifetime in the PAO1 strains co-expressing PvdA-mCherry.

B In PAO1, PvdA is expressed with a much higher number of copies as compared to PvdJ. The choice of the partner labelled with the donor has strong influence on the fraction of donors undergoing FRET $\left(\alpha_{1}\right)$ whereas the $\tau_{1}$ value is mostly unchanged.

Inset: Box-plot of the fraction of pixels better described with a mono-exponential decay in doubly-labelled PvdA/PvdJ bacterial strains. Margins: Histograms of donor only and donor-acceptor fluorescence lifetime $\langle\tau\rangle$.

mega-enzyme acting as a non-ribosomal peptide synthetase (NRPS). The particularity of NRPSs is their ability to produce peptides without the need for the cell ribosomal machinery and messenger RNAs (28). NRPS can build peptides using non-proteinogenic amino-acids. PvdJ is one of the four NRPSs involved in the bio-synthesis of the peptide backbone of the pyoverdine (PVD) precursor (29). Pyoverdine acts as the major siderophore and virulence factor produced by Pseudomonas aeruginosa PAO1 (30). PAO1 pyoverdine contains two hydroxy-ornithines. The presence of a much higher number of PvdA proteins as compared to PvdJ in PAO1 may ensure that the hydroxy-ornithine produced by $\mathrm{PvdA}$ is available in sufficient quantities for PvdJ to insert it in the PVD sequence.

FLIM diagrams of PvdA/PvdJ interactions evidenced strong changes in the values of the amplitude $\alpha_{1}$, depending on which protein was labelled by the donor (Figure 2). If PvdJ is labelled with the donor, a high $\alpha_{1}$ value is observed (in red in Figure 2), together with a very low fraction of pixels that are better described by a mono-exponential fitting model as compared to the two exponential one (box-plot as an in- 

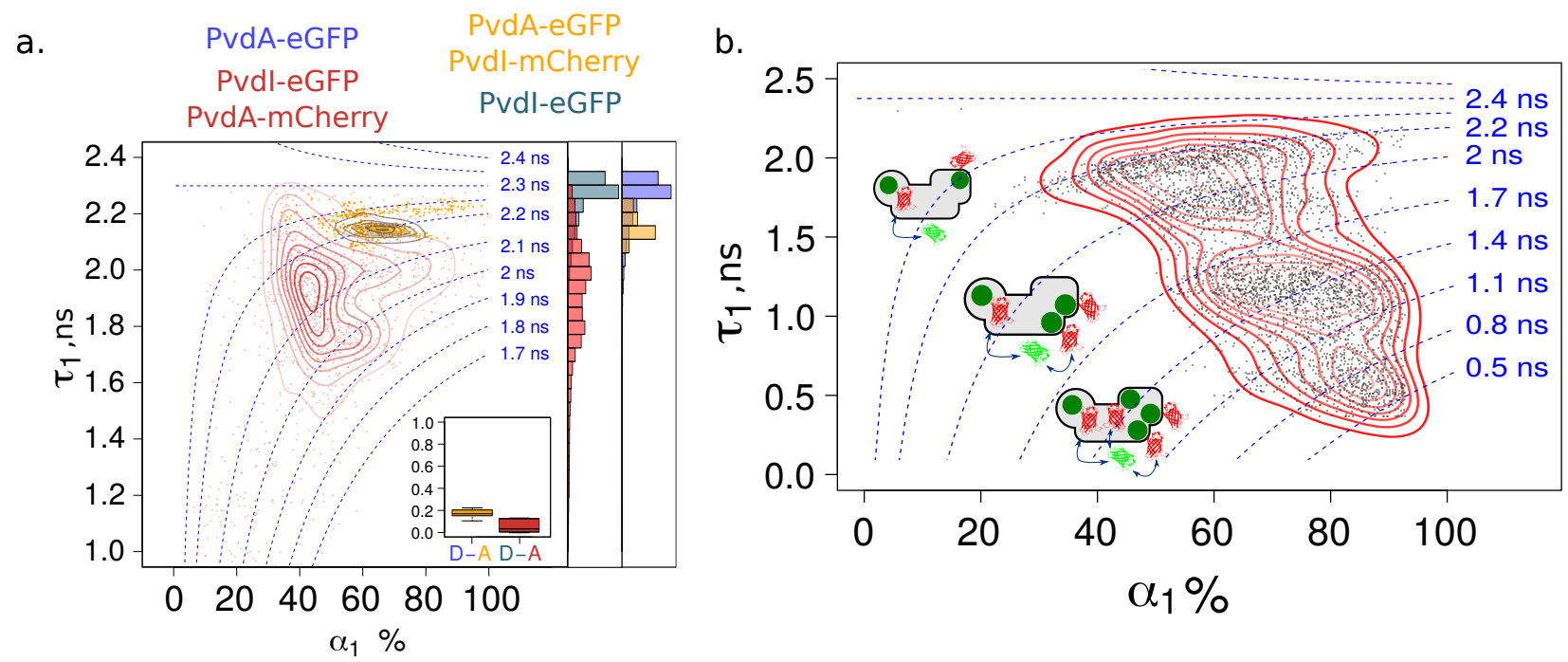

Fig. 3. FLIM diagram in case of FRET with multiple acceptors

a) FLIM diagram of Pvdl-eGFP/PvdA-mCherry (red) and PvdA-eGFP/Pvdl-mCherry (yellow, grey contour) interactions in PAO1. The FLIM parameters of PvdleGFP/PvdA-mCherry (red) largely differed from that of PvdA-eGFP/Pvdl-mCherry FLIM data. $\tau_{1}$ values of Pvdl-eGFP/PvdA-mCherry were scattered and the large distribution of lifetimes indicated the coexistence of complexes with different numbers of acceptors and different FRET efficiencies.

b) Simulation data to explore the effect of multiple acceptors. The presence of multiple acceptors in the complex results in an increased energy transfer and an additional shortening of the donor lifetime.

set in Figure 2). On the opposite, labelling the protein with the higher number of copies with the donor results in similar $\tau_{1}$ value but with a much lower $\alpha_{1}$ distribution (in orange in Figure 2). In addition, the fraction of decays for which a twoexponential model does not statistically improve the quality of fits as compared to a more parsimonious mono-exponential model is about $50 \%$. In sharp contrast to $\alpha_{1}$, the lifetime value of the shortest-lived component $\tau_{1}$ was almost independent on the nature of the protein labelled with the donor and was constant at about $2.0 \mathrm{~ns}$. Taken together, these data indicate that running similar measurements with the fluorescent donor labelling once the less expressed partner and once the most expressed one provides different FLIM parameters when donors and acceptors expression levels are very different. Visualizing and comparing the $\left(\alpha_{1}, \tau_{1}\right)$ distributions of both situations provide an easy analytic tool to prevent misinterpretation on the relative amount of interacting species in the sample.

\section{Complexes with unbalanced stoichiometry}

The FLIM diagram can find even more interesting visualization properties regarding complexes with unbalanced stoichiometry. To illustrate this situation, we explored the interactions of PvdI with PvdA. PvdI is a NRPS involved in the bio-synthesis of pyoverdine in PAO1 together with PvdJ, PvdL and PvdD $(31,32)$. As PvdJ, the expression level of PvdI is very low as compared to that of PvdA (25). Both PvdI and PvdJ are responsible for the addition of a hydroxyornithine in the pyoverdine sequence. PvdI was therefore also likely to harbour PvdA binding sites as PvdJ does. But in sharp contrast to PvdJ/PvdA, the FLIM diagram resulting from FLIM measurement on bacterial strains expressing both eGFP-PvdI and PvdA-mCherry showed a left-skewed distribution with a modal $\tau_{1}$ value of about $2.1 \mathrm{~ns}$ and short $\tau_{1}$ values as small as 1.5ns - 1.6ns (Figure 3). This distribution can be explained by the presence of multiple acceptor in the close vicinity of the eGFP-PvdJ. Indeed, if multiple copies of the acceptor-labelled protein can bind to the donor-labelled protein, an increase in the transfer efficiency occurs with the number of acceptors. The decrease in the fluorescence lifetime of the donor can be calculated as :

$$
\langle\tau\rangle=\left(1 / \tau_{D}+\sum_{i=1}^{n} k_{i} .\right)^{-1}
$$

in which $n$ is the number of acceptors and $k_{i}$ and $\tau_{D}$, the rate of energy transfer and the donor lifetime (assumed here to be unaffected), respectively $(33,34)$. As a consequence, FRET efficiency can be significantly increased if the energy transfer takes place toward multiple acceptors located at fixed distance close to the labelled donor protein. Similarly, reasonable FRET efficiency can be obtained at significantly longer distances than in the case of a single acceptor (35). In most situations, this prevents the value of energy transfer measured by FLIM from being interpreted in terms of distances. In order to better figure-out the effect of multiple acceptors on FLIM parameters, we generated simulated decays corresponding to a mixture situation assuming the presence of up to three acceptors per donor together with a fraction of transfer-free donors (donor lifetime $2.4 \mathrm{~ns}$ ). In addition to a major binding site located $6.5 \pm 1.5 \mathrm{~nm}$ apart from the donor (Förster distance $R_{0}=5.0 \mathrm{~nm}$ ), donor-labelled proteins were allowed to bind acceptors on two additional sites defined at distances of $4.0 \pm 0.5 \mathrm{~nm}$ and $5.0 \pm 1.5 \mathrm{~nm}$ from the donor. As seen on Figure 3, the resulting FLIM diagram resulted in three separable clusters of pixels with lifetimes shortened to about $2 \mathrm{~ns}, 1.2 \mathrm{~ns}$ and $0.5 \mathrm{~ns}$, respectively. These three clusters were corresponding to the acceptor located at the 
major binding site and with the addition of one or two acceptors, respectively, on the additional sites. Interestingly, this pattern was very similar to that observed while exploring the interaction of PvdA with PvdI. This led to the conclusion that multiple PvdA are bound to one molecule of PvdI. This was confirmed by FLIM measurements performed on bacterial strains expressing PvdA-eGFP and PvdI-mCherry (eGFP was labelling the most expressed partner). Using this opposite labelling scheme, the data evidenced the absence of sknewness of $\tau_{1}$ distribution with a central value of $\tau_{1} \approx 2.1$ ns (Figure 3 yellow marginal distribution).

Mechanisms leading to lifetime shortenings are hard to explore based on the sole reading of the $\langle\tau\rangle$ values. Most of the information is given by the distribution of $\tau_{1}$ values. This example demonstrates how the FLIM diagram can be helpful to investigate the mechanisms leading to the shortening of the donor lifetime.

\section{Conclusions}

Multi-exponential decays resulting from FLIM data aiming at characterizing PPIs are difficult to interpret, even when limited to double-exponential decays. One reason comes from the high variability of lifetimes and amplitudes of the individual components resulting from the limited number of photons in the decays of individual pixels. The proposed FLIM diagram plot is a useful visual approach for a more straightforward interpretation of the lifetime data. In this approach, populations of pixels with similar decay signatures can be visualized. By plotting the lifetimes as a function of their amplitudes the density of points reveals central tendencies together with the distribution of the individual parameters. We showed in a few examples that it can be used to provide valuable information about stoichiometry and binding mode of PPIs, even in the presence of large differences in protein expression levels of the two interacting partners. Using the simple experimental strategy of labelling the two interacting partners successively with donor/acceptor and then with acceptor/donor, the graphical representation of the corresponding FLIM data are easy to interpret. Even in situations of large excess of one of the partners, it is relatively easy to discriminate complexes with one-to-one stoichiometry from those with unbalanced stoichiometries. Although helpful, several limitations are not improved by this visualization technique. In particular, the stoichiometry of the complexes cannot be precisely determined and the information remains mostly qualitative. Nevertheless, we believe that multidimensional visualization of FLIM data enhance the usefulness with which FRET experiments in cells can be interpreted for PPIs characterizations.

\section{Material and Methods}

Bacterial strains, plasmids and growth conditions

$P$. aeruginosa strains used in this study are described in (25). Bacteria were grown in $5 \mathrm{~mL}$ lysogeny broth (LB) (Difco) at $30^{\circ} \mathrm{C}$ under $220 \mathrm{rpm}$ orbital shaking. To induce the expression of the pyoverdine pathway enzymes, P. aeruginosa strains were pelleted by centrifugation, washed and further grown overnight at $30^{\circ} \mathrm{C}$ in an iron-deficient succinate medium (composition: $6 \mathrm{gL}^{-1} \mathrm{~K}_{2} \mathrm{HPO}_{4}, 3 \mathrm{gL}^{-1}$ $\mathrm{KH}_{2} \mathrm{PO}_{4}, 1 \mathrm{gL}^{-1}\left(\mathrm{NH}_{4}\right)_{2} \mathrm{SO}_{4}, 0.2 \mathrm{gL}^{-1} \mathrm{MgSO}_{4}, 7 \mathrm{H}_{2} \mathrm{O}$ and $4 \mathrm{~g} \mathrm{~L}^{-1}$ sodium succinate with the $\mathrm{pH}$ adjusted to 7.0 by adding $\mathrm{NaOH}$ ). Cells were finally diluted and grown an additional 24 hours. The presence of pyoverdine in the supernatant can be visually observed as an intense yellow-green water-soluble pigment.

Fluorescence Lifetime Imaging Microscopy (FLIM)

Time-correlated single-photon counting FLIM measurements were performed on a home-made two-photon excitation scanning microscope based on an Olympus IX70 inverted microscope with an Olympus $60 \times 1.2 \mathrm{NA}$ water immersion objective operating in de-scanned fluorescence collection mode $(10,36)$. Two-photon excitation at $930 \mathrm{~nm}$ was provided by a Ti:Sapphire oscillator (Insight ${ }^{\circledR}$ DeepSee ${ }^{\mathrm{TM}}$, Spectra Physics - $80 \mathrm{MHz}$ repetition rate, $\approx 70 \mathrm{fs}$ pulse width) at $10-20 \mathrm{~mW}$. Fluorescence photons were collected through a $680 \mathrm{~nm}$ short pass filter (F75-680, AHF, Germany) and a 525/50 nm bandpass filter (F37-516, AHF, Germany) and directed to a fibrecoupled avalanche photo-diode (SPCM-AQR-14-FC, Perkin Elmer) connected to a time-correlated single photon counting (TCSPC) module (SPC830, Becker \& Hickl, Germany). Cells grown for $48 \mathrm{~h}$ in succinate media were immobilised on a $1 \%$ agarose pad and rapidly imaged. Typically, area of $50 \times 50 \mu \mathrm{m}$ in the samples were scanned at $4 \mu$ s per pixel $(1024 \times 1024$ pixels) for $100 \mathrm{~s}$ to $600 \mathrm{~s}$ to reach the NyquistShannon sampling criteria and to achieve the appropriate photon statistics to investigate the fluorescence decays. Fluorescence decays were processed using a commercial software package (SPCImage V2.8, Becker \& Hickl, Germany). A binning of $5 \times 5$ pixels was applied before processing the fluorescence decays. FLIM data were further analysed using a homemade ImageJ plugin and R scripts.

Software and Hardware Availability A R package named flimDiagRam for Fluorescence Lifetime Imaging data analysis is available on https://github.com/jgodet/flimDiagRam. Most figures of this article can be reproduced independently using the code found on this repository.

\section{ACKNOWLEDGEMENTS}

We acknowledge Dr Ludovic Richert for his valuable assistance on FLIM data acquisition and for the technical maintenance and development of the FLIM setup. We thank Dr. Isabelle Schalk at University of Strasbourg for providing bacteria strain constructions expressing fluorescent labelled proteins. This work was funded by grants from Fondation pour la Recherche en Chimie (https://icfrc.fr/). YM is grateful to the Institut Universitaire de France (IUF) for support and providing additional time to be dedicated to research.

\section{AUTHOR CONTRIBUTIONS}

These contributions follow the Contributor Roles Taxonomy guidelines: https://casrai.org/credit/. Conceptualisation: J.G, Y.M; Data curation: J.G.; Formal analysis: J.G.; Funding acquisition: J.G., Y.M.; Investigation: J.G.; Methodology: J.G.; Project administration: J.G.; Resources: J.G., Y.M.; Software: J.G.; 
Supervision: Y.M.; Validation: J.G., Y.M.; Visualisation: J.G.; Writing - original draft: J.G.; Writing - review \& editing: J.G., Y.M..

\section{COMPETING FINANCIAL INTERESTS}

The authors have declared that no conflicting interests exist

\section{Bibliography}

1. Jones S and Thornton J M 1996 Proceedings of the National Academy of Sciences of the United States of America 93 13-20 ISSN 0027-8424 URL http://www.pnas.org/cgi/doi/10.1073/pnas.93.1.13http://ww . ncbi.nlm.nih.gov/pubmed/8552589http://www.pubmedcentral.nih. gov/articlerender. fCgi?artid=PMC40170

2. Nooren I M A and Thornton J M 2003 The EMBO journal 22 3486-92 ISSN 02614189 URL http://www.ncbi.nlm.nih.gov/pubmed/12853464http://www. pubmedcentral.nih.gov/articlerender.fcgi?artid=PMC165629

3. Duncan R R, Bergmann A, Cousin M A, Apps D K and Shipston M J 2004 Journal of microscopy 215 1-12 ISSN 0022-2720 URL http://www.ncbi.nlm.nih.gov/ pubmed/15230870http://www. pubmedcentral.nih.gov/articlerender. fCgi?artid=PMC1903372

4. Chen Y, Mills J D and Periasamy A 2003 Differentiation; research in biological diversity 71 528-41 ISSN 0301-4681 URL http://www.ncbi.nlm.nih.gov/pubmed/ 14686950

5. Peter M and Ameer-Beg S M 2004 Biology of the cell 96 231-6 ISSN 0248-4900 URL http://www.ncbi.nlm.nih.gov/pubmed/15182705

6. Duncan R R 2006 Biochemical Society transactions 34 679-82 ISSN 03005127 URL http://www.ncbi.nlm.nih.gov/pubmed/17052173http://www. pubmedcentral.nih.gov/articlerender.fcgi?artid=PMC1855982

7. Festy F, Ameer-Beg S M, Ng T and Suhling K 2007 Molecular bioSystems 3 381-91 ISSN 1742-206X URL http://www.ncbi.nlm.nih.gov/pubmed/17533451

8. Wallrabe H and Periasamy A 2005 Current opinion in biotechnology 16 19-27 ISSN 09581669 URL http://www.ncbi.nlm.nih.gov/pubmed/15722011

9. Fritz J V, Dujardin D, Godet J, Didier P, De Mey J, Darlix J L, Mély Y and de Rocquigny H 2010 Journal of Virology 84 1585-1596 ISSN 1098-5514 URL http: // www. ncbi.nlm. nih.gov/pubmed/19923179

10. El Meshri S E, Dujardin D, Godet J, Richert L, Boudier C, Darlix J L, Didier P, Mély Y and de Rocquigny H 2015 Journal of Molecular Biology 427 1480-1494 ISSN 00222836 URL http://www.ncbi.nlm.nih.gov/pubmed/25644662https:// linkinghub.elsevier.com/retrieve/pii/s002228361500037

11. Margineanu A, Chan J J, Kelly D J, Warren S C, Flatters D, Kumar S, Katan M, Dunsby C W and French P M W 2016 Scientific reports 628186 ISSN 20452322 URL http://www.ncbi.nlm.nih.gov/pubmed/27339025http://www. pubmedcentral.nih.gov/articlerender.fcgi?artid=PMC4919659

12. Meyer-Almes F J 2017 Methods and applications in fluorescence 5042002 ISSN 2050-6120 URL http://www.ncbi.nlm.nih.gov/pubmed/28660853

13. Köllner M and Wolfrum J 1992 Chemical Physics Letters 200 199-204 ISSN 00092614 URL https://linkinghub.elsevier.com/retrieve/pii/000926149287068z

14. Knutson J R, Beechem J M and Brand L 1983 Chemical Physics Letters 102501 507 ISSN 00092614 URL https:// linkinghub.elsevier.com/retrieve/pii/ 0009261483874545

15. Beechem J M, Gratton E, Ameloot M, Knutson J R and Brand L 2002 The Global Analysis of Fluorescence Intensity and Anisotropy Decay Data: Second-Generation Theory and Programs Topics in Fluorescence Spectroscopy (Boston, MA: Springer US) pp 241-305 URL http://link.springer.com/10.1007/0-306-47058-6\{_\}5

16. Verveer P J, Squire A and Bastiaens P I 2000 Biophysical journal 78 2127-37 ISSN 0006-3495 URL http://www.ncbi.nlm.nih.gov/pubmed/10733990http: //www . pubmedcentral. nih.gov/articlerender. fcgi?artid=PMC1300804

17. Menezes F, Fedorov A, Baleizão C, Valeur B and Berberan-Santos M N 2013 Methods and applications in fluorescence 1015002 ISSN 2050-6120 URL http://www. ncbi.nlm. nih.gov/pubmed/29148435

18. Fereidouni F, Blab G A and Gerritsen H C 2014 Methods and applications in fluorescence 2035001 ISSN 2050-6120 URL http://www.ncbi.nlm.nih.gov/pubmed/ 29148469

19. Fereidouni F, Gorpas D, Ma D, Fatakdawala $H$ and Marcu L 2017 Methods and applications in fluorescence 5035003 ISSN 2050-6120 URL http://www.ncbi.nlm.nih.gov/ pubmed/28644150http://www. pubmedcentral.nih.gov/articlerender. fCgi?artid=PMC 6043162

20. Silberberg M and Grecco H E 2017 Methods and applications in fluorescence 5024016 ISSN 2050-6120 URL http://www.ncbi.nlm.nih.gov/pubmed/28649965

21. Le Marois A, Labouesse S, Suhling K and Heintzmann R 2017 Journal of Biophotonics 10 1124-1133 ISSN 1864063X URL http://doi.wiley.com/10.1002/jbio. 201600160

22. Becker $W$ The bh TCSPC Handbook, 7th edn.(Becker \& Hickl GmbH 2019) URL https://www.becker-hickl.com/wp-content/uploads/2019/01/ hb-bh-TCSPC.pdf

23. Richert L, Didier P, de Rocquigny $H$ and Mély $Y 2015$ Monitoring HIV-1 Protein Oligomerization by FLIM FRET Microscopy pp 277-307 URL http:// link. springer.com/10. 1007/978-3-319-14929-5\{_\}8

24. Vogel S S, Thaler C and Koushik S V 2006 Science's STKE : signal transduction knowledge environment 2006 re2 ISSN 1525-8882 URL http:// www. ncbi.nlm.nih.gov/ pubmed/16622184

25. Gasser V, Malrieu M, Forster A, Mély Y, Schalk I J and Godet J 2019 bioRxiv 438713 URL http://biorxiv.org/content/early/2019/04/05/438713.abstract

26. Meneely K M, Barr E W, Bollinger J M and Lamb A L 2009 Biochemistry 48 4371-6
ISSN 1520-4995 URL http://www.ncbi.nlm.nih.gov/pubmed/19368334http: //www pubmedcentral, nih gov/articlerender, fcgi?artid=PMC2710847

27. McMorran B J, Shanta Kumara H M, Sullivan K and Lamont I L 2001 Microbiology (Reading, England) 147 1517-24 ISSN 1350-0872 URL http://www.ncbi.nlm.nih.gov/ pubmed/11390682

28. Süssmuth R D and Mainz A 2017 Angewandte Chemie - International Edition 56 3770-3821 ISSN 15213773

29. Visca P, Imperi F and Lamont I L 2007 Trends in microbiology 15 22-30 ISSN 0966-842X URL http://linkinghub.elsevier.com/retrieve/pii/ S0966842X06002678http://www.ncbi.nlm.nih.gov/pubmed/17118662

30. Schalk I J and Guillon L 2013 Environmental microbiology 15 1661-73 ISSN 1462-2920 URL http://www.ncbi.nlm.nih.gov/pubmed/23126435

31. Lehoux D E, Sanschagrin F and Levesque R C 2000 FEMS microbiology letters 190 141-6 ISSN 0378-1097

32. Gasser V, Baco E, Cunrath O, August P S, Perraud Q, Zill N, Schleberger C, Schmidt A, Paulen A, Bumann D, Mislin G L A and Schalk I J 2015 Environmental microbiology n/a-n/a ISSN 1462-2920 URL http: // doi . wiley.com/10.1111/1462-2920. 13199http://www.ncbi.nlm.nih.gov/pubmed/26718479

33. Lakowicz J R 2006 Principles of fluorescence spectroscopy (Springer) ISBN 9780387312781 URL http://books. google.fr/books?id=-PSybuLNxcAC

34. Bunt $G$ and Wouters F S 2017 Biophysical reviews 9 119-129 ISSN 18672450 URL http://www.ncbi.nlm.nih.gov/pubmed/28424742http://www. pubmedcentral.nih.gov/articlerender.fcgi?artid=PMC5380710

35. Bojarski P, Kulak L, Walczewska-Szewc K, Synak A, Marzullo V M, Luini A and D'Auria S 2011 The journal of physical chemistry. B 115 10120-5 ISSN 1520-5207 URL http: //www.ncbi.nlm.nih.gov/pubmed/21780798

36. Batisse J, Guerrero S X, Bernacchi S, Richert L, Godet J, Goldschmidt V, Mély Y, Marquet R, de Rocquigny H and Paillart J C 2013 Journal of virology 87 6492-506 ISSN 1098-5514 URL http: // www. ncbi.nlm.nih. gov/pubmed/23576497http: //www.pubmedcentral.nih.gov/articlerender.fcgi?artid=PMC3648125 
Supplementary Note 1: FLIM data simulation 


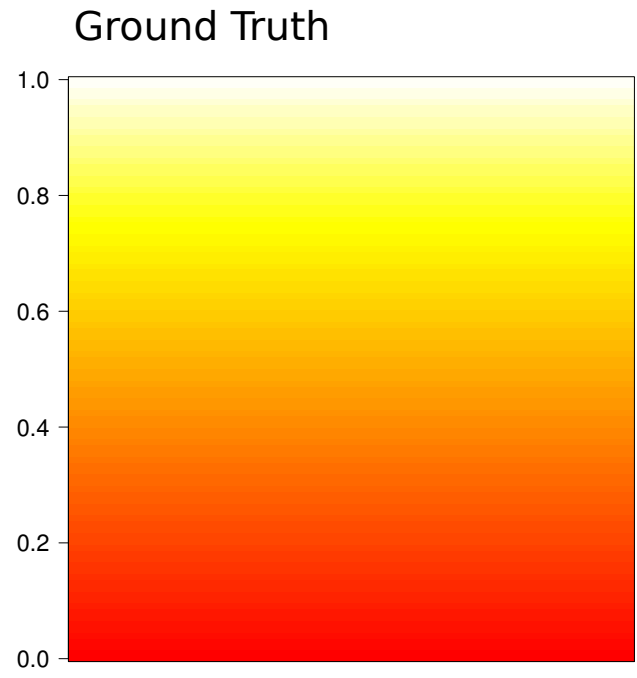

$$
\begin{aligned}
& \tau_{1}=2.0 \mathrm{~ns} \\
& \tau_{2}=2.4 \mathrm{~ns}
\end{aligned}
$$

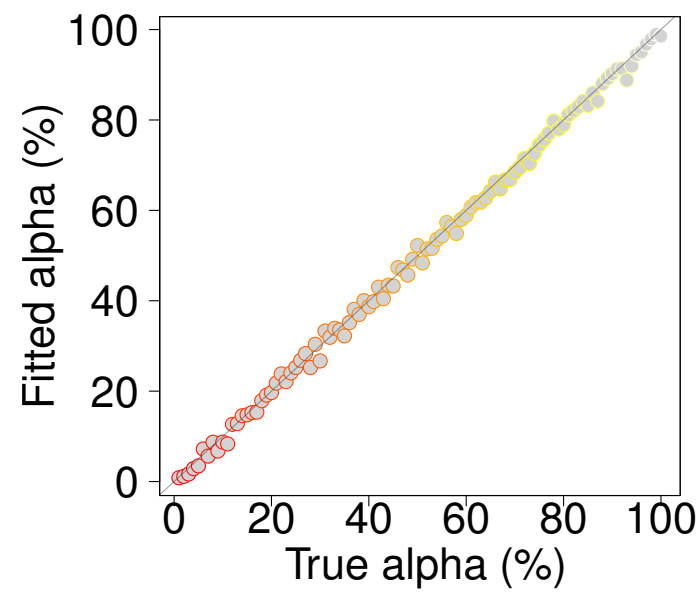

\section{Fitted data}

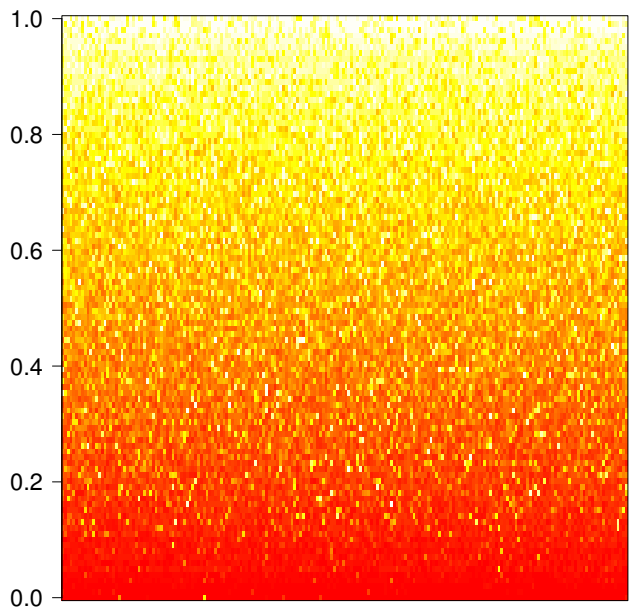

$\tilde{\tau_{1}}=2.00 \mathrm{~ns} \quad \mathrm{IQR}=[1.90,2.07]$

$\tau_{2}$ fixed at $2.4 \mathrm{~ns}$

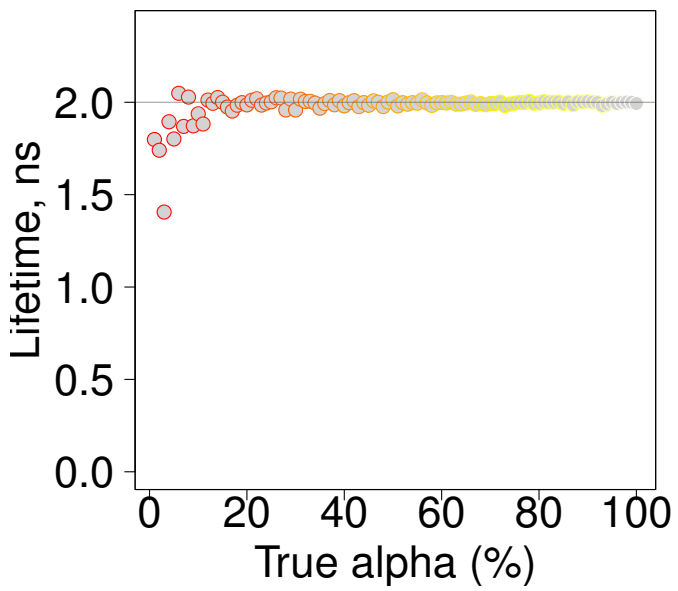

Fig. S1. Fitting procedure validation. Results achieved from simulated fluorescence lifetime decay curves indicate that the $\tau_{1}$, fixed $\tau_{2}, \alpha_{1}$, and $\alpha_{2}$ recovered after fitting from simulated decay curves at low SNR are concordant with their ground truth. $\alpha$ values are accurate at $\pm 2 \%$ and $\tau_{1}$ value is precisely retrieved as soon as $\alpha$ is a least $20 \%$, even for a situation in which $\tau_{1}$ is only shortened by $1 / 6$ as compared to the value of $\tau_{2}$ (2 ns vs $\left.2.4 \mathrm{~ns}\right)$. 